\title{
Inverter Parallelization for an Islanded Microgrid Using the Hopf Oscillator Controller Approach with Self-synchronization Capabilities
}

\author{
Mingshen Li, Student Member, IEEE, Yonghao Gui, Senior Member, IEEE, Yajuan Guan, Member, IEEE, \\ Jose Matas, Josep M. Guerrero, Fellow, IEEE, and Juan C. Vasquez, Senior Member, IEEE
}

\begin{abstract}
Nonlinear dynamical systems such as weakly coupled oscillators are an interesting approach to be adopted for the regulation of power inverters inside microgrids. Aiming at the synchronization and load sharing in islanded mirogrid, this paper is inspired by oscillator synchronization property to propose a Hopf oscillator controller for the single-phase inverters. The Hopf oscillator dynamic equations are used for providing the inverter's frequency and amplitude voltage references which lead to a robust nonlinear droop behavior for driving the system without using communications. The Hopf oscillator provides better sharing of the load between inverters with higher robustness, less harmonic distortion, and faster time response of the associated limit cycle than the achieved by the other approach made with a Van der Pol oscillator. In addition, global asymptotic synchronization of system is proven by Lyapunov approach. Simulation results of a system composed by paralleled inverters are provided and compared with a Van der Pol oscillator approach reported in literature. Experimental results are also provided to prove the Hopf oscillator based controller under different circumstances.
\end{abstract}

Index Terms-Paralleled single-phase inverters, Microgrid, Hopf oscillator control, synchronization, current sharing.

\section{INTRODUCTION}

$\mathbf{M}$ ICROGRID, has acted an effective and critical role in highly distributed power systems, which generally include islanding and grid-connecting operation modes. In AC microgrids, as the interface from distributed sources to electric network, the inverter can be designed and controlled to satisfy different requirements such as power sharing, grid synchronization, and power quality improvement, etc. For islanding situation, the decentralized control are principally preferred due to its operation flexibility and non-communication requirments. Therefore, the conventional droop control was widely used in the last decade, which aims to share power for

This work was funded by Center for Research on Microgrid Villum Investigator grant (No.25920) from The Villum Foundation, and also Industrial cooperation project (YBN2018085257) supported.

Mingshen. Li, Yajuan. Guan, J. M. Guerrero, and J. C. Vasquez are with the Dept. of Energy Technology, Aalborg University, 9220 Aalborg, Denmark (e-mail: (msh; ygu; joz; juq)@et.aau.dk). Yonghao Gui is with the Dept. of Electronic System, Aalborg University, 9220 Aalborg, Denmark (e-mail: yg@es.aau.dk) Jose Matas Alcala is with the Dept. of Electronic Engineering, Polytechnic University of Catalonia, 08800 Barcelona, Spain (e-mail: jose.matas@upc.edc) emulating the synchronous generator though building a linear relationship between active and reactive powers with respect to the inverter's frequency and voltage [1], [2]. Although there are many modified methods to improve, the drawbacks are still obvious such as: slow dynamic response, active and reactive powers coupling, impressionable performance with line impedance and nonlinear loads etc [3]. In [4], a synchronousreference frame virtual impedance loop-based controller was proposed to conquer above mentioned, but the applied Phaselocked-loop (PLL) will limit the control bandwidth and the virtual impedance is complex to design.

Theoretically, nonlinear oscillators have a very interesting property, namely the synchronization due to their weak interaction which means the coupling relationship can only have slight effects on oscillator dynamics over a period. These interaction could lead to phase locking and frequency synchronization in the network [5]. For last 30 years, oscillators have been investigated to use in biological modeling such as neuronal signaling models and beat perception; to engineering modelling such as robot control and image processing [6]-[8]. A series of studies which apply the oscillator synchronization characteristic to parallel inverters control have been carried out recently in [9]-[14], the non-communication method is known as virtual oscillator control (VOC). The motivation of VOC is inspired by the coupling dynamics of weakly nonlinear oscillators based on the Liénard's Theorem, and the strategy as a time-domain controller can be designed as a inner loop which distinguishes with the phasor domain of droop control. In contrast with droop control, VOC exhibits some advantages: faster transient response, independency to loads and without hierarchical control. In [9], [10], [12], the dead-zone oscillators were employed in VOC, which decomposes the virtual current source into a dead-zone. In order to improve the dynamics and simplify the piecewise function, the Van der Pol oscillator was introduced into the micro-controller in [11], [13], [14]. These works demonstrated the nonlinear droop relationship between active power and terminal voltage, and reactive power and frequency. In addition, the small-signal stability of VOCcontrolled inverters was analyzed in [15]. Nevertheless, the salient drawbacks of dead zone and Van der Pol oscillator controller can be summarized as follows:

1) A strict sufficient condition for synchronization should be guaranteed, which means it permits phase-locking depending on the stronger coupling strength [16]; 
2) An extra limit cycles constraint is necessary to design according to Liénard's Theorem. Dead zone and Van der Pol oscillator are typical harmonics oscillators [17], and parameters should meet a certain condition if the ideal sinusoidal is to be obtained. Generally, an approximate limit circle in phase is selected as the references, which certainly affect the reference precision. Hence, the additional harmonics are generated in the controller;

3) The initial states affect the stability of system and the evolution speed from the initial state to the steady state [18].

However, some nonlinear oscillators such as PoincaréBendixson (PB) and Hopf oscillator can produce ideal limit cycle which can not be easily affected by initial states and can recover from external perturbation in a short time [19]. In [20], an adaptive frequency synchronization method based on the PB oscillator is proposed for converters, which offers high robustness against grid faults. Recently, a dispatchable virtual oscillator control (dVOC) is proposed in [21]-[23] to mimic coupled Hopf oscillators system, and the method makes power set-points dispatch to realize grid-connected mode application. However, these works primarily focus on the power dispatchability, and there is little effort on the internal synchronization investigation for islanded mode particularly the application in single-phase systems. In addition, it lacks of the oscillator performance analysis compared with the oscillators proposed before.

To cope with the issues of the previous controllers that are highlighted above, a reliable and simple controller is proposed to mimic the coupled Andronov-Hopf oscillators systems, which only consists of a voltage loop. We apply the Hopf oscillator dynamics to parallel single-phase inverters system with the output current feedback. In contrast with the droop controller, there are no active and reactive power calculation, low pass filter, and PLL in the proposed controller. To compare with VOC, the proposed controller doesn't need to consider limit cycle constraint and the strict sufficient synchronization condition, and presents the better power quality and the simpler parameters designing. In brief, the main contributions of this paper are listed below:

1) provides a theoretical comparison with Van der Pol oscillator regarding the harmonics analysis, robust performance, and synchronization dynamics;

2) presents a modified Hopf oscillator controller for parallel single phase inverters, and derives averaged model to contribute the droop characteristic analysis;

3) proves the global asymptotic synchronization without the tight sufficient condition;

4) validates the effectiveness of proposed controller both in simulation and experiments.

The organization of this paper is presented as follows. In section II, the dynamics of the Hopf oscillator are described by the following second-order differential equations and we draw a the property comparison between the Hopf and Van der Pol oscillators in terms of the harmonics, the robustness and the synchronization performance under the same situations. Section III presents the Hopf oscillator controller implementation details and its averaged model is derived, also the parameters selection is discussed according to designing requirement. The
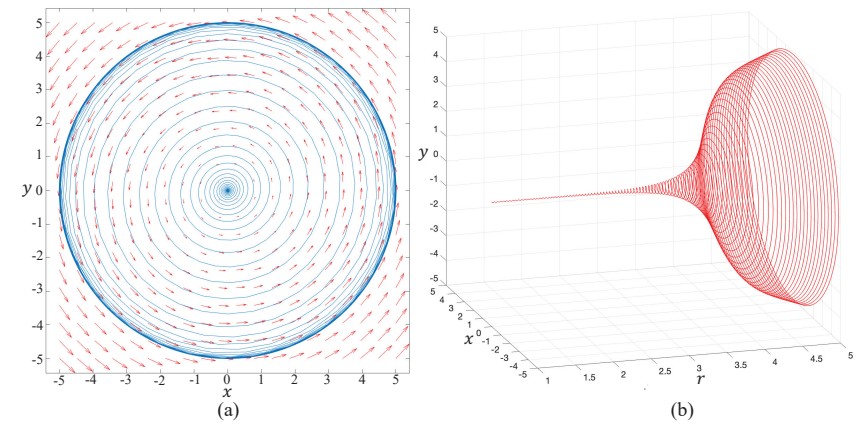

Fig. 1. (a) Vector field of the Hopf oscillator in phase plane; (b)3-D dynamics of Hopf oscillator with $r$ changing

global asymptotic synchronization of coupled Hopf oscillators is proved in Section IV. Section V and VI show the simulation and experimental results that validates the effectiveness of the proposed controller. Finally, the conclusion and future works are outlined in Section VII.

\section{DYNAMICS OF HOPF OSCILLATOR}

\section{A. Hopf Oscillator Introduction}

The ordinary dynamics of the Hopf oscillator can be described by the following second-order differential equations:

$$
\left\{\begin{array}{l}
\dot{x}=\mu\left(r^{2}-x^{2}-y^{2}\right) x+\omega y \\
\dot{y}=\mu\left(r^{2}-x^{2}-y^{2}\right) y-\omega x .
\end{array}\right.
$$

Where $x$ and $y$ are the orthogonal states, $\mu$ is is the model coefficient that affects the time transient response speed of the oscillator, $r$ refers to the output amplitude in steady-state, and $\omega$ is the oscillation frequency. The stable periodic solutions of Hopf oscillator are:

$$
x=r \sin \left(\omega t+\theta_{0}\right), y=r \cos \left(\omega t+\theta_{0}\right) .
$$

Where $\theta_{0}$ is determined by the initial state. Fig. 1(a) represents the the vector field and series of trajectories in the phase plane from initial condition $(0,0)$, which helps to visualize the direction and intensity of the flow. In the steady state, a close orbit circle is generated where the solutions of system are orthogonal signals. Fig. 1(b) shows the 3-D dynamics diagram when the parameter $r$ is changed from 1 to a non-null value [24]. It indicates that the behavior of Hopf oscillator is characterized by an damping oscillation with continues amplitude variation, and the stable limit cycles maintain oscillations if $r>0$.

\section{B. Comparison of Van der Pol Oscillator and Hopf Oscil- lator}

The Van der Pol oscillator is a classical relaxation oscillator that is employed in VOC [11]- [14]. In order to compare with Hopf oscillator, the property two types of oscillators are discussed in this part. The Van der Pol oscillator dynamics can be written in a 2-dimensional form:

$$
\left\{\begin{array}{l}
\dot{x}=y \\
\dot{y}=\rho\left(1-x^{2}\right) y-\omega^{2} x .
\end{array}\right.
$$


Where $\rho$ is the model coefficient which determines the degree of relaxation part of the system; $\omega$ influences the frequency of the oscillations, and $\rho$ also influences the frequency.

1) Harmonics Analysis: First, the limit cycle comparison of two type oscillators is introduced with the both coefficients step changing. As shown in Fig. 2(a), the limit cycle evolution of Van der Pol oscillator becomes increasingly sharp with increasing $\rho$. Note that the limit cycles evolve to cycles for $\rho<0.1$ with fix amplitude equal to 2 and $\omega=100 \pi$, which means the states output are periodic sinusoidal form. Thus, the Van der Pol oscillator became a simple harmonic oscillator. By contrast, in Fig. 2(b), the limit cycles evolve to the same circle with different $\mu$ when oscillaton frequency is $100 \pi$, and $\mu$ only affects the damping speed [25]. Therefore, there is a strict parameter constraint on the Van der Pol oscillator to be an ideal circular, but the damping coefficient is free for Hopf oscillator.

For deriving the harmonic solutions for Hopf oscillator, the Poincaré-Lindstedt method [26], [27] is employed to approximate the limit cycle. The simplified second order equation of the Hopf oscillator with a new time variable $\tau=\omega t$ can be given by:

$$
\omega^{2} x^{\prime \prime}+x=\varepsilon \mu \omega\left(r^{2}-x^{2}-x^{\prime 2}\right) x^{\prime}=\varepsilon f(x)
$$

where $\varepsilon$ formally stands for a small parameter. The natural time scale of the oscillations can be expended as:

$$
\begin{aligned}
& x_{\varepsilon}(\tau)=x_{0}(\tau)+\varepsilon x_{1}(\tau)+\ldots \\
& \omega_{\varepsilon}=\omega_{0}+\varepsilon \omega_{1}+\ldots \\
& f(x, \varepsilon)=f_{1}(x)+\varepsilon f_{2}(x)+\ldots
\end{aligned}
$$

Substituting (5) to (4), the equations up to second order in terms of $\varepsilon$ can be obtained:

$$
\begin{aligned}
& x_{0}^{\prime \prime}+x_{0}=0 \\
& x_{1}^{\prime \prime}+x_{1}=f_{1}\left(x_{0}\right)-2 \omega_{1} x_{0}^{\prime \prime} \\
& x_{2}^{\prime \prime}+x_{2}=f_{2}\left(x_{0}\right)+\frac{\partial f_{1}\left(x_{0}\right)}{\partial x_{0}} x_{1}-2 \omega_{1} x_{1}^{\prime \prime}-\left(\omega_{1}^{2}+2 \omega_{2}\right) x_{0}^{\prime \prime}
\end{aligned}
$$

Suppose the initial sate $x_{0}(0)=a$, and it is clear that the zero order is a simple oscillation with the solution $x_{0}(\tau)=$ $a \cos \tau$. Substituting the zero order solution to the first order and second order equations, the first order equation is given by:

$$
x_{1}^{\prime \prime}+x_{1}=a \mu\left(a^{2}-r^{2}\right) \sin \tau+2 \omega_{1} a \cos \tau
$$

In order to avoid the secular terms, select $a=r, \omega_{1}=$ 0 . Thus, solution of first order equation is $x_{1}(\tau)=a \cos \tau$. Similarly, the second order equation is given by:

$$
\begin{array}{r}
x_{2}^{\prime \prime}+x_{2}=a \varepsilon \mu\left(a^{2}-r^{2}\right) \sin \tau+a \mu\left(a^{2}-r^{2}\right) \cos \tau \\
+2 \omega_{1} \cos \tau+\left(\omega_{1}^{2}+2 \omega_{2}\right) \cos \tau
\end{array}
$$

For eliminating the resonant forces of equation right side, select $a=r, \omega_{1}=0, \omega_{2}=0$. The solution of second order equation is also $x_{2}(\tau)=\operatorname{acos} \tau$. Thus, the approximation solution of Hopf oscillator oscillate in the fundamental frequency $\omega_{0}$.

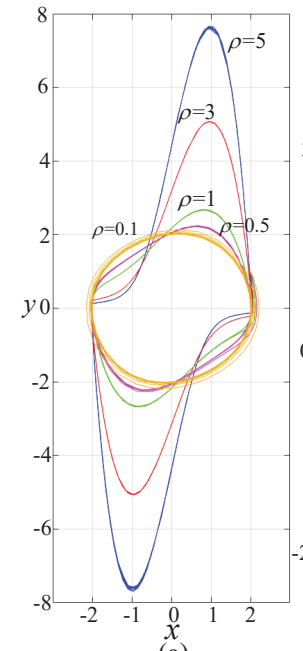

(a)

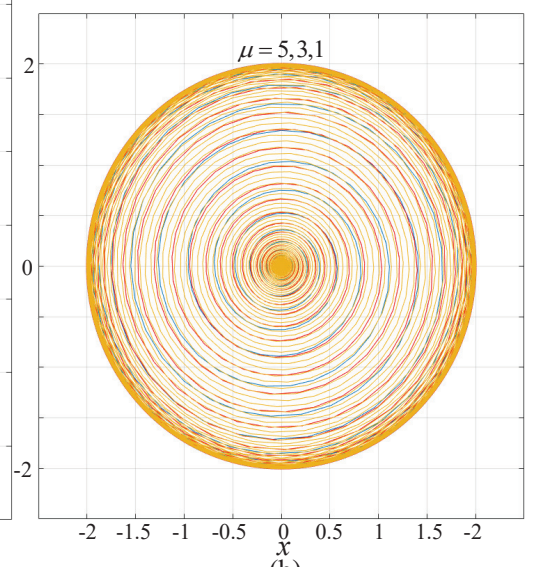

(b)
Fig. 2. The limit cycles evolution when parameter varies in the phase plane: (a)Van der Pol oscillator. (b) Hopf oscillator with $\omega=100 \pi$.

In the same way, the first order expansion for Van der Pol oscillator can be given by [28]:

$$
x_{1}^{\prime \prime}+x_{1}=2 a \omega_{1} \cos \tau+a\left(0.25 a^{2}-1\right) \sin \tau+0.25 a^{3} \sin 3 \tau
$$

For eliminating the resonant forces, select $\omega_{1}=0, a=2$, the solution of first order is $x_{1}(\tau)=\sin ^{3} \tau$. Note that if $a$ is small enough, the right side is approximately zero, in which the solution of oscillator is $x_{1}(\tau) \approx a \cos \tau$. Considering the second order, there is [28]:

$$
x_{2}^{\prime \prime}+x_{2}=\left(4 \omega_{2}+11\right) \cos \tau-31 \cos ^{3} \tau+20 \cos ^{5} \tau
$$

The approximate solution is $x_{2}(\tau)=2 \cos \tau+\rho \sin ^{3} \tau+\ldots$ with $\omega=1-0.0625 \rho^{2}+\ldots$. Therefore, the solution of Van der Pol oscillator contains harmonics whose amplitudes are directly proportional to $\rho$.

In summary, the Van der Pol oscillator is a harmonic oscillator, and its approximate solution expansion mainly includes three-order harmonics. By contrast, the hopf oscillator has an orbitally exponentially stable solution with the fundamental frequency.

2) Robust Performance: Next, we focus on the robust performance of two oscillators in steady sate. The Van der Pol and Hopf oscillators oscillate in sinusoidal with the same frequency, amplitude and initial state $(0.1,0)$. The reference error responses under unit impulse and 0.1p.u. step are shown in Fig. 3. As shown in Fig. 3 (a), the Hopf oscillator represents a faster recovery, but the Van der Pol oscillator eliminates error slowly under the unit impulse of amplitude. Similarly, the step response of Hopf is faster than Van der Pol as shown in Fig. 3 (b).

3) Synchronization Performance: At last, the coupling synchronization performance comparison of two types oscillator are discussed. The synchronization occurs due to the mutual interaction in coupling oscillators system, which is usually described using the Kuramoto model. For a fair comparison, the two oscillators oscillate at the same limit cycle with 
(a)

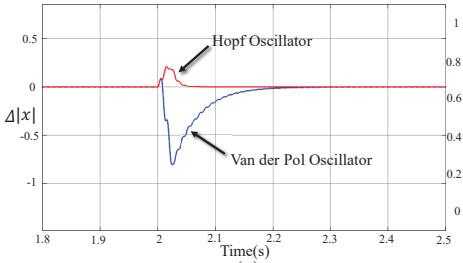

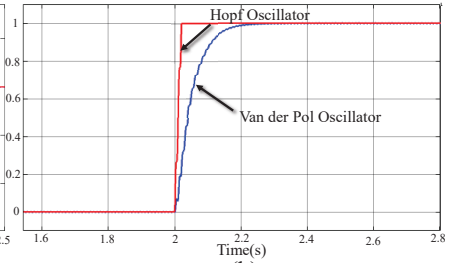

(b)
Fig. 3. The dynamics response of amplitude error comparison under (a) the unit impulse and (b) the step.

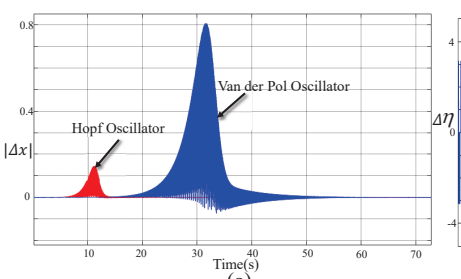

(a)

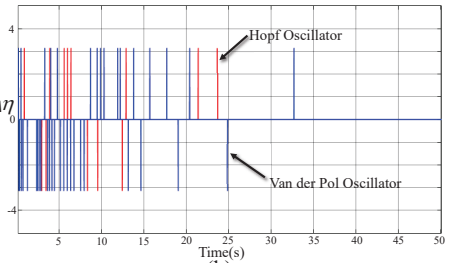

(b)
Fig. 4. The state error dynamics between the coupled oscillators for two types oscillators:(a) the amplitude error (b) the phase error.

$r=1$ and $\omega=100 \pi$, and definition of the coupling strength is $\gamma=0.25$. Accordingly, the coupling error equations of amplitude and phase in steady state are defined as:

$$
\begin{array}{r}
|\Delta x|=\gamma\left(\left|x_{1}\right|-\left|x_{2}\right|\right) \\
\Delta \eta=\gamma\left(\eta_{1}-\eta_{2}\right) .
\end{array}
$$

Where $\left|x_{1}\right|,\left|x_{2}\right|, \eta_{1}$ and $\eta_{2}$ are the amplitude and phase of two coupling oscillators in steady state, respectively.

The synchronization trends of the amplitude and phase difference for Van der Pol and Hopf oscillators are shown in Fig. 4 (a) and (b). For their both, the systems start from different initial conditions and arrive to full synchronization at the end. By contrast, it is clear that hopf oscillator provides faster amplitude and phase transient responses and smaller amplitude overshoot.

As a conclusion, the limit cycles of Hopf oscillator are an ideal circular for $r>0$ while the Van der Pol oscillator has the strict parameters constraint to be an ideal circular. The dynamic responses under disturbance and the synchronization speed of Hopf are faster than Van der Pol's under the same operating situation.

\section{Controller Implementation ANd PARAmeter DESIGN}

In this section, the proposed Hopf-oscillator controller for paralleled single-phase inverters is introduced, and the controller parameters are designed. In addition, the averaged model is derived to observe the relationship between active and reactive power outputs and the inverter output voltage dynamics.

\section{A. Controller Implementation}

The Hopf oscillator controller implementation for parallel single-phase inverters is depicted in Fig. 5. The physical system includes a DC source, a full-bridge inverter, and an

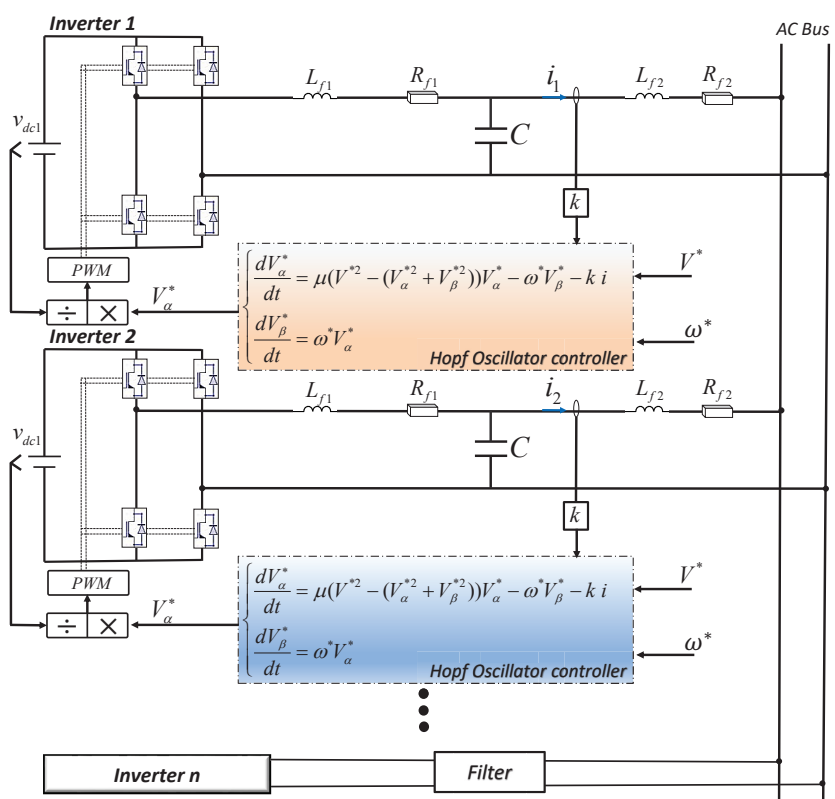

Fig. 5. Control structure the proposed method.

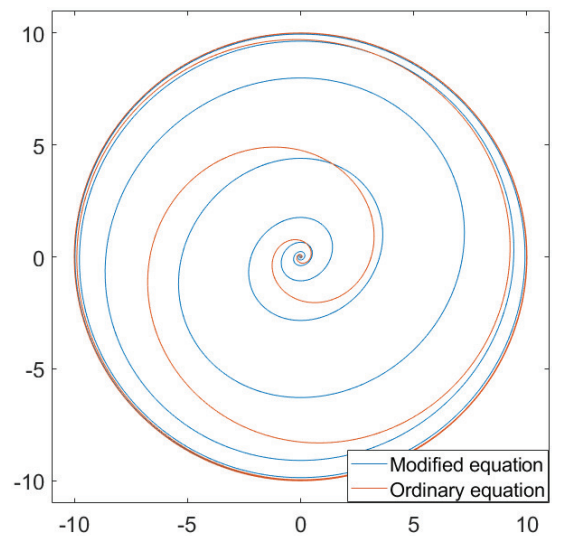

Fig. 6. The limit cycle evolution comparison of Hopf oscillators with $r=$ $10, \omega=100 \pi$.

LCL filter consisting of inverter-side inductor $L_{f 1}$ and resistor $R_{f 1}$, and output inductor $L_{f 2}$ and resistor $R_{f 2}$. Furthermore, the time-domain controller only contains a voltage inner loop with the scaled feedback current, which is measured from inverter output side. According to the amplitude reference $V^{*}$ and resonant frequency $\omega^{*}$, the orthogonal state signals $V_{\alpha}$ and $V_{\beta}$ in the $\alpha-\beta$ frame are generated by Hopf oscillator, and the state $V_{\alpha}$ is selected as the voltage reference for singlephase application. In order to decouple the dc-link dynamics, the pulse width modulation (PWM) of the voltage reference is divided by the dc-link voltage.

The simplified dynamics of Hopf oscillator controller in continuous time-domain based on (4) are described by the following differential equations:

$$
\left\{\begin{array}{l}
\dot{V}_{\alpha}=\mu\left(V^{* 2}-V_{\alpha}^{2}-V_{\beta}^{2}\right) V_{\alpha}-\omega V_{\beta}-k i \\
\dot{V}_{\beta}=\omega V_{\alpha}
\end{array}\right.
$$

Where $V_{\alpha}, V_{\beta}$ are the states of the oscillator, $i$ indicates 
the output current as external periodic perturbation, $k$ is the feedback gain and $V^{*}$ determines steady state amplitude voltage $\left(V_{\alpha \infty}^{2}+V_{\beta \infty}^{2}=V^{* 2}\right)$.

Note that $\mu\left(r^{2}-x^{2}-y^{2}\right)$ of (1) is oscillation magnitude correction part which converges to zero in the phase plane. From the limit cycle evolution viewpoint, the simplified oscillator (12) and ordinary Hopf oscillator (1) with same initial condition $(0,0)$ are compared in Fig. 6. It can be seen that they can reach the same steady state despite the mismatch of the limit cycle evolution. Consider the one state is used as voltage reference modulation in single-phase inverter system, so the other magnitude part $\mu\left(r^{2}-x^{2}-y^{2}\right) x$ can be ignored from ordinary equations.

In order to apply the controller to a practical situation, (12) is discretized by employing the trapezoidal rule for computing integrals, and the discretization of Hopf controller can be presented as follow:

$$
\left\{\begin{array}{l}
\left.V_{\alpha}(z)=V_{\alpha}(z) z^{-1}+0.5 T_{s} M(z)\left(1+z^{-1}\right)\right) \\
V_{\beta}(z)=V_{\beta}(z) z^{-1}+0.5 T_{s} \omega\left(V_{\alpha}(z)+V_{\alpha}(z) z^{-1}\right)
\end{array}\right.
$$

Where $T_{s}$ donates the sampling time, and $M(z)=\mu\left(V(z)^{* 2}-\right.$ $\left.V_{\alpha}(z)^{2}-V_{\beta}(z)^{2}\right) V_{\alpha}(z)-\omega V_{\beta}(z)-k i(z)$.

Therefore, the discrete equations are able to be implemented into a digital controller.

\section{B. Averaged Model of Hopf Oscillator Controller}

Since the performance of the nonlinear differential equation in time scale is difficult to be observed, the averaged model is employed to obtain the approximated periodic solutions for the voltage amplitude and phase through replacing a vector field by its average.

As we are interested in the phase dynamics, the system is written in polar coordinated. By differentiating $V_{\alpha}=V \sin (\theta)$, $V_{\beta}=V \cos (\theta)$ with respect to time, the phase dynamics of Hopf oscillator based on (12) are obtained as follows:

$$
\left\{\begin{array}{l}
\dot{V}=\mu\left(V^{* 2} V-V^{3}\right) \cos ^{2}(\theta)-k i \cos (\theta) \\
\dot{\theta}=\omega-\mu\left(V^{* 2}-V^{2}\right) \sin (\theta) \cos (\theta)+\frac{k i}{V} \sin (\theta) .
\end{array}\right.
$$

Where $\theta$ is the instantaneous phase angle of the inverter output. Subsequently, the average value of a periodic signal $V(t)$ in the period $T$ is presented by:

$$
\bar{V}=\frac{1}{T} \int_{0}^{T} V(t) d t
$$

In order to simplify the averaged model [11], the dynamics of the inverter terminal voltage is defined as:

$$
\frac{d \theta}{d t}=\omega+\frac{d \varphi}{d t}=\omega^{*}+\frac{d \varphi^{*}}{d t}
$$

Where $\omega$ and $\omega^{*}$ are the nominal frequency of the inverter outputs and the steady state frequency of inverter output, respectively. The angles $\varphi$ and $\varphi^{*}$ indicate the phase offset with respect to $\omega$ and $\omega^{*}$, respectively. Therefore, in the averaged model, the single Hopf oscillator dynamical system under $2 \pi$-periodic function in time domain are presented as follows:

$$
\left\{\begin{array}{l}
\dot{\bar{V}}=\frac{\omega^{*}}{2 \pi} \int_{0}^{\frac{2 \pi}{\omega^{*}}} \mu\left(V^{* 2} V-V^{3}\right) \cos \left(\omega^{*} t+\varphi^{*}\right)^{2} d t \\
-\frac{\omega^{*} k}{2 \pi} \int_{0}^{\frac{2 \pi}{\omega^{*}}} i \cos \left(\omega^{*} t+\varphi^{*}\right) d t \\
\dot{\bar{\varphi}}^{*}=-\frac{\omega^{*}}{2 \pi} \int_{0}^{\frac{2 \pi}{\omega^{*}}} \mu\left(V^{* 2}-V^{2}\right) \sin \left(\omega^{*} t+\varphi^{*}\right) \\
\cos \left(\omega^{*} t+\varphi^{*}\right) d t+\frac{\omega^{*} k}{2 \pi V} \int_{0}^{\frac{2 \pi}{\omega^{*}}} i \sin \left(\omega^{*} t+\varphi^{*}\right) d t .
\end{array}\right.
$$

Based on (17), the averaged dynamics of the amplitude and phase of one oscillator are expressed as:

$$
\left\{\begin{array}{l}
\dot{\bar{V}}=\frac{V^{* 2} \mu}{2} \bar{V}-\frac{\mu}{2} \bar{V}^{3}-\frac{k}{\bar{V}} \bar{P} \\
\dot{\bar{\theta}}=\omega^{*}-\omega+\frac{k}{\bar{V}^{2}} \bar{Q} .
\end{array}\right.
$$

\section{Parameter Selection}

Given the dynamics of the Hopf oscillator controller in (12), parameters $\mu$ and $k$ show the significant impact on the system performance. According to the oscillator dynamics principle, $\mu$ has impact on the damping performance regarding the relaxation speed, and $k$ is a coupling coefficient that is considered as a feedback gain. In addition, the initial conditions of the oscillator also determine the transient response as mentioned before, so define the two initial conditions for integral as $V_{\alpha 0}$ and $V_{\beta 0}$. Therefore, how to design these parameters in parallel inverters is discussed.

1) Damping Coefficient $\mu$ : Define the evolution time of oscillator as $t_{\text {rise }}$ that determines the unloaded inverter achieves its reference voltage from initial conditions. Assume the controller operates in open-circuit when $\bar{P}=0$, and the voltage rises from $0.1 \bar{V}$ and $0.9 \bar{V}$. We define $W=\bar{V}^{2}$ and $W_{\text {ref }}=V^{* 2}$. Based on (18), the evolution time can be expressed as:

$$
t_{\text {rise }}=\frac{1}{\mu} \int_{0.01 W_{\text {ref }}}^{0.81 W_{\text {ref }}} \frac{1}{W W_{\text {ref }}-W^{2}} d W=\frac{6.045}{\mu V^{* 2}}
$$

Notice that the evolution time is inversely proportional to the voltage amplitude references and $\mu$. Therefore, the designer can obtain the desired transient time by performing a proper selection of $V^{*}$ and $\mu$.

2) Initial States of the Oscillator: In theoretical perspective, the coupled Hopf oscillators always converge to a limit cycle wherever the initial states locate [5]. Fig. 7 (a) illustrates the trajectories of two coupled Hopf oscillator with different initial states, and it can be seen that their orbits towards asymptotically limit cycle with fixed amplitude voltage and frequency. However, considering the converging speed of system and safe startup in practice, it is preferred to select the initial state as $\left(0.5 V_{\text {rate }}, 0\right)$ where $V_{\text {rate }}$ is the rated voltage of inverter.

3) Current Coefficient $k$ : First, we need to analyze the bifurcation diagram to detect the critical conditions that happen when $k$ are varied from zero to 7000. It means for (12), whether changing $k$ will cause a damping of the oscillation amplitude. Fig. 7 (b) shows the 3-D diagram with $k$ varying for an oscillator with fixed frequency $50 \mathrm{~Hz}$ and amplitude $185 \mathrm{~V}$. It can be seen that the limit cycle of Hopf oscillator presents the persistent closed orbit under the large margin of $k$ changing. 


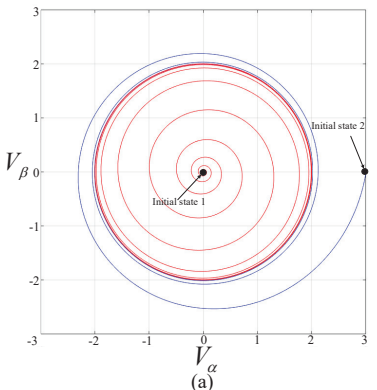

(a)

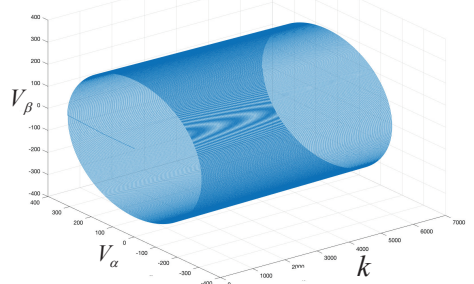

(b)
Fig. 7. The impacts of current gain and initial states on system dynamics: (a)3-D diagram of feedback oscillator with $k$ varying; (b)The trajectories for two Hopf oscillators converging to the same limit cycle with different initial states $(0,0)$ and $(3,0)$

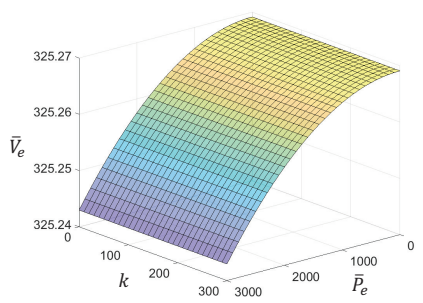

(a)

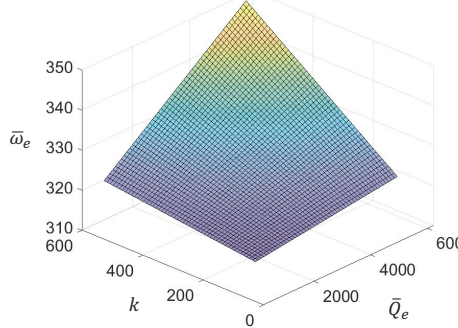

(b)
Fig. 8. (a) The relationship between $k$ and the equilibrium voltage amplitude and active power. (b) The relationship between $k$ and the equilibrium frequency and reactive power

Therefore, one oscillator is able to keep stable with maintained oscillations considering the varying feedback parameters.

To investigate the voltage equilibrium point of the system (18), the following equation needs to be considered:

$$
V^{* 2} \mu \bar{V}_{e}^{2}-\mu \bar{V}_{e}^{4}-2 k \bar{P}_{e}=0 .
$$

Where $\bar{V}_{e}$ and $\bar{P}_{e}$ refers to the equilibrium voltage amplitude and average active power in steady state respectively. In this paper, the positive roots of (20) can be expressed as:

$$
\bar{V}_{e}=\sqrt{0.5 V^{* 2} \pm 0.5 \sqrt{V^{* 4}-\frac{8}{\mu} k \bar{P}_{e}}}
$$

The two real roots can be obtained if the $k$ satisfies:

$$
0<k \leq k_{c}=\frac{\mu V^{* 4}}{8 \bar{P}_{e}} .
$$

From (22), the critical value $k_{c}$ is proportional to $V^{* 4}$ and inversely proportional to the equilibrium averaged active power. Fig. 8 illustrate the nonlinear droop law of $V-P, \omega-Q$. For the voltage regulation as shown in Fig. 8 (a), parameter $k$ is insensitive to regulate output voltage. Meanwhile, the frequency value is proportional to the reactive power in Fig. 8 (b), and the maximum frequency offset should regulate the frequency variation.

To facilitate the parameters design, define the rated power of inverter $P_{\text {rated }}$ as the equilibrium active power, so $k_{c}$ is given by:

$$
k_{c}=\frac{\mu V^{* 4}}{8 P_{\text {rated }}}
$$

Moreover, given $i_{t h}$ and $j_{t h}$ inverters with terminal voltages $v_{i}$ and $v_{j}$, and active powers $P_{i}$ and $P_{j}$, respectively, synchronized $i_{t h}$ and $j_{t h}$ inverters can be expressed as $v_{i}=v_{j}$ in steady state. According to (18), the amplitude's integration of two oscillators can be equal at steady state, and the relationship between power rating and $k$ is presented as follow:

$$
\frac{P_{i}}{P_{j}}=\frac{k_{j}}{k_{i}} .
$$

In this case, assume that the filter impedance of each inverter are identical. However, in real cases, the design of the filter not only affects the power quality of the system but also has impact on the power rating.

\section{GLOBAL ASYMPTOTIC SYNCHRONIZATION OF COUPLED OSCILLATORS}

It is essential to derive the sufficient conditions on coupled Hopf oscillators dynamical systems that realize synchronization. In this section, the exact conditions for linearly coupled Hopf oscillators are derived by using Lyapunov approach [29] [30].

Using the state coordinates definition initialed for the Hopf oscillator in the section, the differential equations for $N$ linearly coupled Hopf oscillators can be given as:

$$
\left\{\begin{array}{l}
\dot{x}_{i}=\mu\left(r_{i}^{2}-x_{i}^{2}-y_{i}^{2}\right) x_{i}-\omega y_{i}-\frac{k}{Z_{L}} \sum_{p=1}^{N}\left(x_{p}-x_{i}\right) \\
\dot{y}_{i}=\omega x_{i} \quad \forall i=1, \ldots N .
\end{array}\right.
$$

Where $Z_{L}$ is the common load for the system. The definition of synchronization for the oscillators can be expressed as:

$$
\lim _{t \rightarrow \infty}\left(x_{j}-x_{i}\right)=0, \lim _{t \rightarrow \infty}\left(y_{j}-y_{i}\right)=0, \quad \forall j, i=1, \ldots N .
$$

Therefore, according to (25) and (26), the derivative of synchronization is given by:

$$
\left\{\begin{array}{l}
\dot{x}_{j}-\dot{x}_{i}=\mu\left(r_{j}{ }^{2}-x_{j}^{2}-y_{j}^{2}\right) x_{j}-\mu\left(r_{i}{ }^{2}-x_{i}^{2}-y_{i}^{2}\right) x_{i} \\
-\omega\left(y_{j}-y_{i}\right)-\frac{k N}{Z_{L}}\left(x_{j}-x_{i}\right) \\
\dot{y}_{j}-\dot{y}_{i}=\omega\left(x_{j}-x_{i}\right) .
\end{array}\right.
$$

As mentioned in Section II, the Hopf oscillator has strong robustness performance and closed orbit is stable. Assume in steady state $x_{i \infty}^{2}+y_{i \infty}^{2}=r^{2}$ under feedback perturbation $k i$ and each oscillator has same frequency. In order to obtain the sufficient synchronization of coupling gain $k$, Theorem 1 is proposed to establish synchronization.

Theorem 1: Consider coupled oscillators system dynamics (25), for variables $x_{i}, x_{j}, y_{i}, y_{j} \in \mathbf{R}, \forall j, i=1, \ldots N$., as $t \rightarrow$ $\infty$, the coupled Hopf oscillators synchronize asymptotically, if the parameters $k>0$.

Proof: Define the Lyapunov function $S_{i j}$ function as:

$$
S_{i j}=\frac{1}{2}\left(x_{j}-x_{i}\right)^{2}+\frac{1}{2}\left(y_{j}-y_{i}\right)^{2} .
$$


TABLE I

SYSTEM PARAMETERS USED IN SIMULATION AND EXPERIMENT

\begin{tabular}{ccc}
\hline \hline Parameter & Value & Unit \\
\hline DC voltage & 450 & $\mathrm{~V}$ \\
Rated Power & 2.2 & $\mathrm{~kW}$ \\
Filter inductance & 1.8 & $\mathrm{mH}$ \\
Filter capacitance & 25 & $\mu \mathrm{F}$ \\
Resistive load & 180 & $\Omega$ \\
Step-up load & 180 & $\Omega$ \\
Oscillator initial state & 155,0 & $\mathrm{~V}, \mathrm{~V}$ \\
Startup $k$ & 600 & $\mathrm{~A} / \mathrm{A}$ \\
Damping Coefficient $\mu$ & 5 & $V^{-2} s^{-1}$ \\
Voltage reference & 311 & $\mathrm{~V}$ \\
Frequency reference & 50 & $\mathrm{~Hz}$ \\
\hline \hline
\end{tabular}

By differentiating (28), the Lyapunov function can be expressed as:

$$
\begin{aligned}
\dot{S_{i j}=} & -\frac{k N}{Z_{L}}\left(x_{j}-x_{i}\right)^{2}+\mu x_{j}\left(x_{j}-x_{i}\right)\left(r_{j}^{2}-x_{j}^{2}-y_{j}^{2}\right) \\
& -\mu x_{i}\left(x_{j}-x_{i}\right)\left(r_{i}^{2}-x_{i}^{2}-y_{i}^{2}\right) x_{i} \\
& -\omega\left(x_{j}-x_{i}\right)\left(y_{j}-y_{i}\right)+\omega\left(x_{j}-x_{i}\right)\left(y_{j}-y_{i}\right) .
\end{aligned}
$$

As $x_{i \infty}^{2}+y_{i \infty}^{2}=r_{i}{ }^{2}, x_{j \infty}^{2}+y_{j \infty}^{2}=r_{j}{ }^{2}$ is mentioned before, (29) is simplified as follows:

$$
\dot{S_{i j}}=-\frac{k N}{Z_{L}}\left(x_{j}-x_{i}\right)^{2} .
$$

Based on (30), it is can be concluded that $\dot{S}_{i j}$ will be negative if $k>0$. Set $X$ as the largest invariant aggregate for $\dot{S}_{i j}=0$ where there exists $x_{j}(t)-x_{i}(t)=0, y_{j}(t)-y_{i}(t)=$ $0, \dot{x}_{j}(t)-\dot{x}_{i}(t)=0$ and $\dot{y}_{j}(t)-\dot{y}_{i}(t)=0$ of each oscillator. According to Lasalle's invariance principle, all trajectories $\dot{x}_{j}(t)-\dot{x}_{i}(t)$ and $\dot{y}_{j}(t)-\dot{y}_{i}(t)$ are able to converge to $X$ when $t \rightarrow \infty$. Therefore, the coupled oscillators synchronize asymptotically.

\section{SIMULATION RESULTS}

In order to validate the performances of the proposed Hopfoscillator controller, the Virtual Van der Pol oscillator has been chosen to compare. The two parallel inverters based on two types of oscillators have been simulated with MATLAB/SIMULINK. The control algorithm of Hopf oscillator is based on (12) with discrete integrators, and the control theme and parameters of VOC are used from [11]. The proposed controller parameters are chosen by Table. I. Various simulation tests have been carried out, such as: startup, connection, current ratio changes, THD of unload voltage and large variation of load power.

\section{A. Startup and Connection}

Fig. 9. shows the transient response of output current and active power with the scenario of VSI \#1 startup and VSI \#2 connection in the presence of a resistive load. As seen in Fig. 9 (a)(b), the Van der Pol oscillator controller has approximately $0.8 \mathrm{~s}$ starting time, and the settling time is $0.1 \mathrm{~s}$ when VSI \#2 connects. Note that the initial conditions of Van der Pol oscillators were selected to be $3 \mathrm{~V}$ considering emulating errors. In comparison, the starting time of the Hopf oscillator

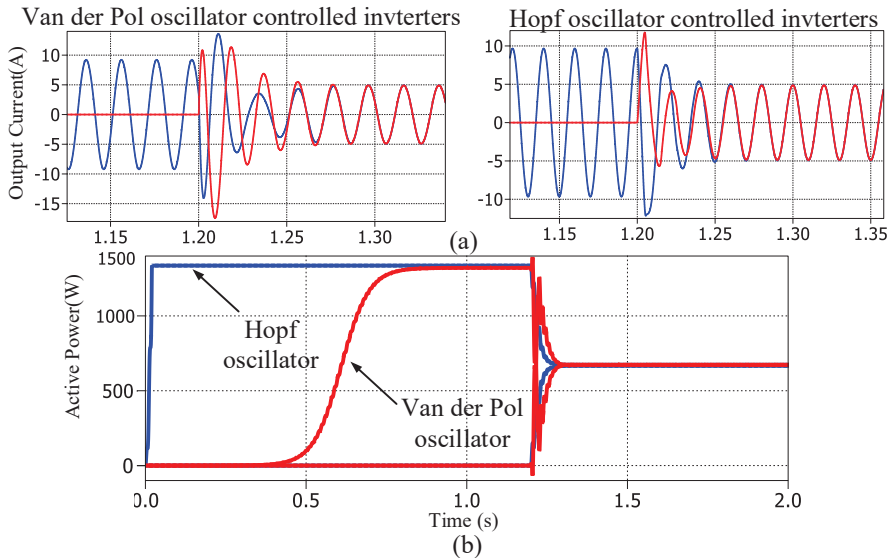

Fig. 9. Comparison simulation results of inverter output current and active power with startup and connection.

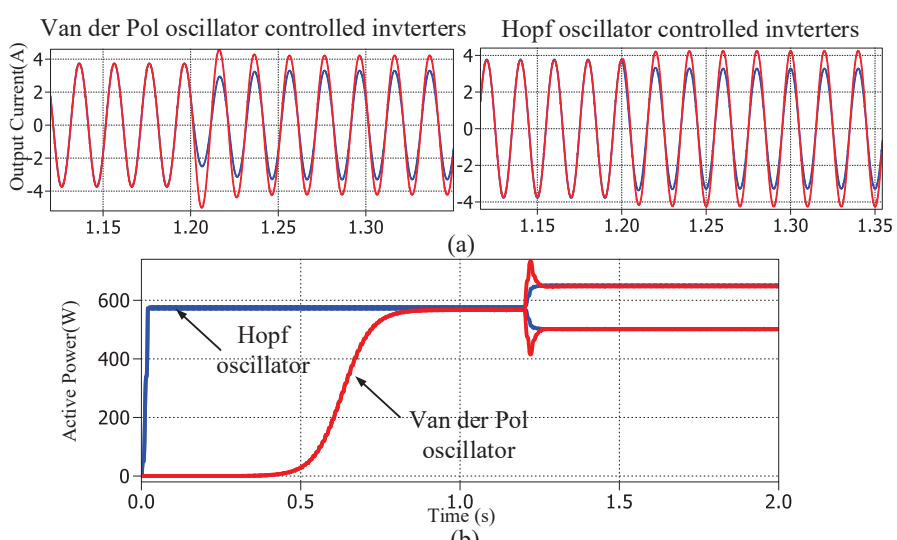

(b)

Fig. 10. Comparison simulation results of inverter output current and active power when current ratio changes.

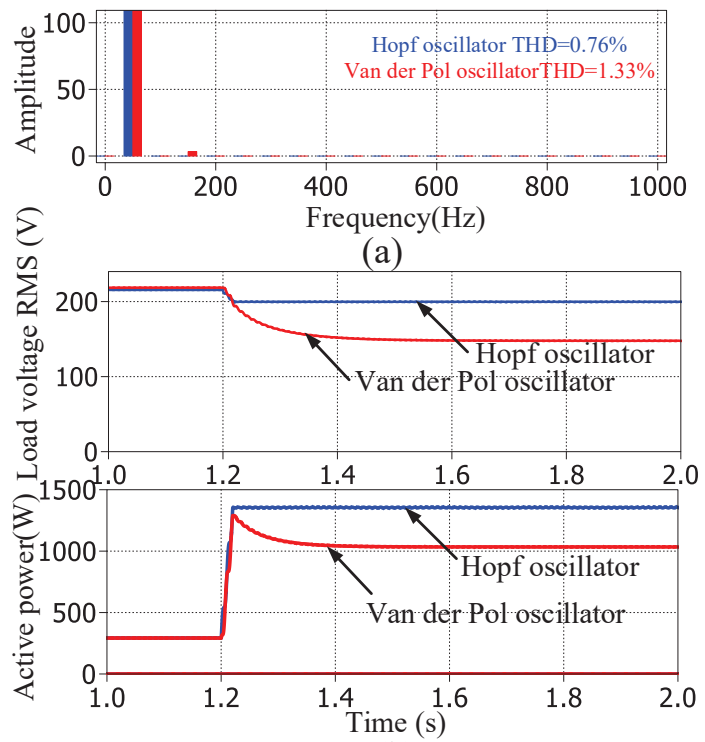

(b)

Fig. 11. Comparison simulation results of (a) Voltage THD of unloaded inverter and (a) the large variation of load power. 
controller is approximately $0.02 \mathrm{~s}$ with $3 \mathrm{~V}$ initial conditions. The obvious difference is because the evolution time (19) is smaller then the evolution time of $\operatorname{Van} \operatorname{der} \operatorname{Pol}\left(t_{\text {rise }}=\frac{6}{\omega^{*} \varepsilon \sigma}\right)$ [11]. The proposed controller is able to realize power sharing and the settling time of connection is approximately $0.06 \mathrm{~s}$. It is clear to observe that the Hopf oscillator reaches steady state faster than the Van der Pol in case of startup and connection.

\section{B. Ratio Changes}

The transient response for sudden direct currents ratio changes of parallel VSIs with two control strategies are illustrated in Fig. 10 (a)(b). The current ratio has been suddenly changed from 1:1 to $1: 2$. It can be observed that the current outputs of two control methods is nearly instantaneous from 1:1 to 1:2. Note that the transient response of Hopf oscillator controller lasts $0.045 \mathrm{~s}$ compared with $0.04 \mathrm{~s}$ of the Van der Pol.

\section{Unload Voltage THD and Large Load Power Variation}

At first, the voltage harmonic content of unload inverter is shown in Fig. 11 (a). The total harmonic distortion (THD) of Hopf and Van der Pol controller is $0.76 \%$ and $1.33 \%$, respectively. The few third-order harmonics are included in the terminal voltage from Van der Pol controller which agrees the harmonics analysis in Section.II. Considering the large load power variation case, the a oscillator-controlled inverter supplies the resistive load with power $P_{1}=280 \mathrm{~W}$ in steady state at the beginning, then a large load power $P_{2}=5 P_{1}=$ $1.4 k W$ is switched to system at $1.2 \mathrm{~s}$. The transient responses comparison of load voltage RMS and active power are shown in Fig. 11 (b). Notice that the load voltage of the proposed method has a smaller decrease with $9 \%$ of rated voltage while Van der Pol controlled inverter has $32 \%$ voltage drops. Thus, the proposed controller achieves better performance under the large load power variation.

\section{EXPERIMENTAL RESULTS}

The performance of the Hopf-oscillator control is also validated by three parallel single phase inverters in experimental platform where the setup and its configuration are shown in Fig. 12. The prototype consists of the DC voltage supply source, the three Danfoss inverters with LCL filters, and the resistive load and nonlinear load. The inverters' voltage and current are measured by the DS2004 A/D board and also captured by oscilloscope. Each inverter operates at $10 \mathrm{kHz}$ switching frequency, and the control algorithm is implemented in the dSPACE DS1006 real-time system where the differential equations (13) were implemented by $0.1 \mathrm{~ms}$ sampling step. The setup and controller parameters are listed in Table. I.

\section{A. Inverters Connection}

At first, we consider the inverters connection case when each inverter connects one by one to supply a common resistive load. For observing the connecting performance with different initial state, we set oscillator initial state of inverter

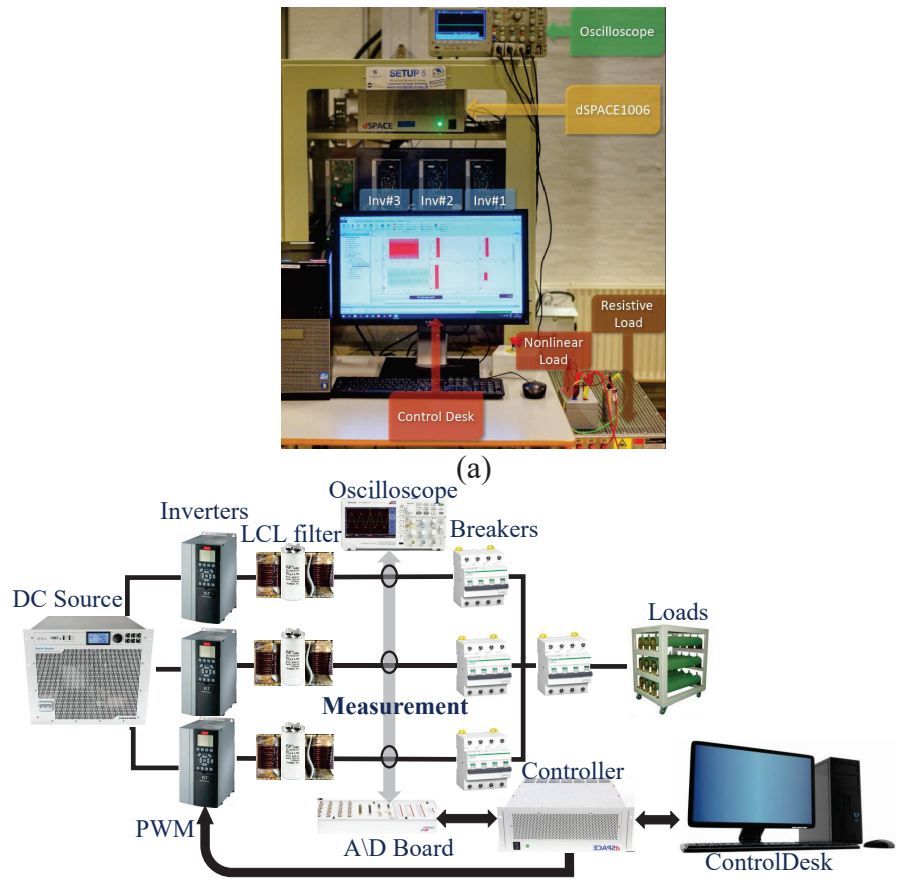

(b)

Fig. 12. (a)Experimental setup in the laboratory.(b)Configuration of experiment.

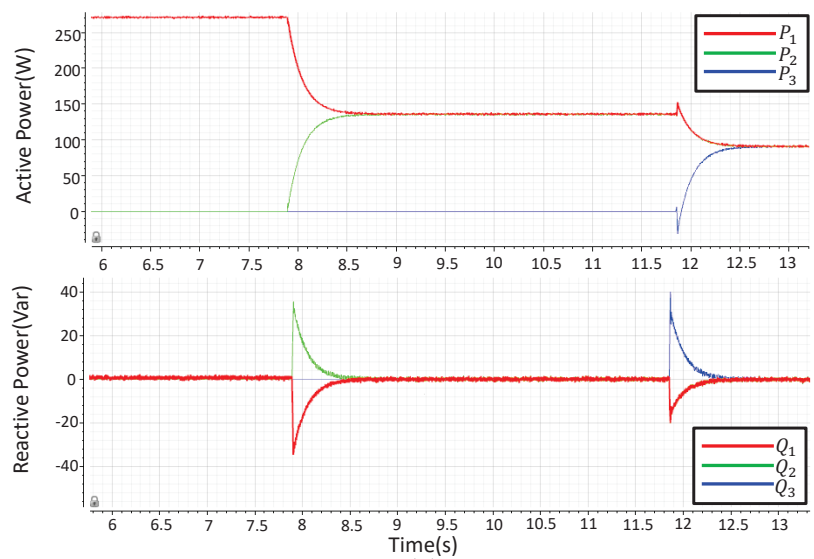

(a)

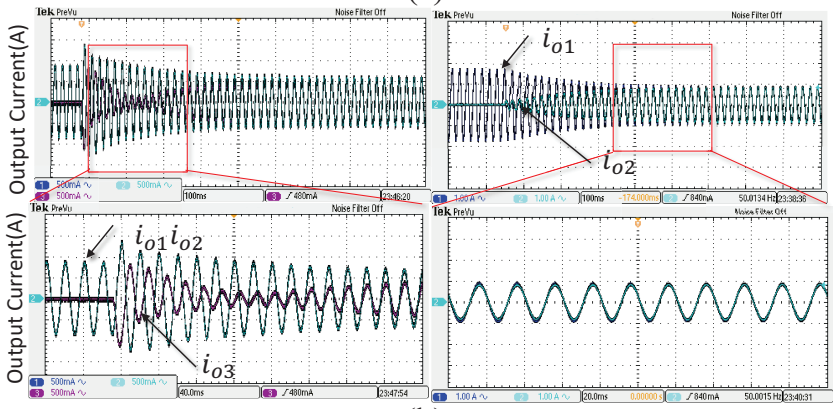

(b)

Fig. 13. Transient response of three inverters connection one by one.(a)Instantaneous active and reactive power.(b)Output current of inverter \#1,\#2 and \#3. (c) Load voltage. 

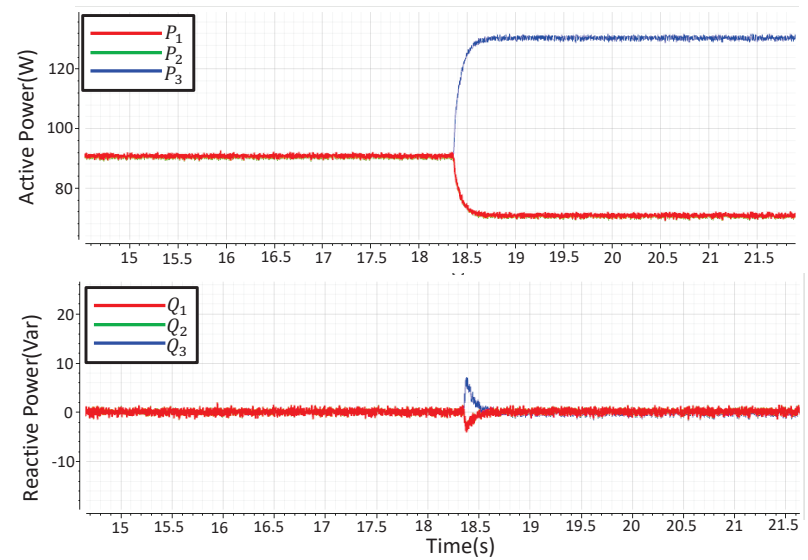

(a)

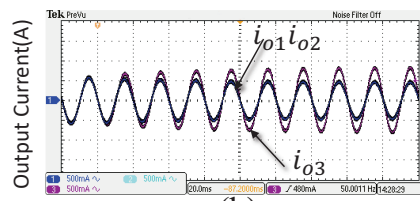

(b)

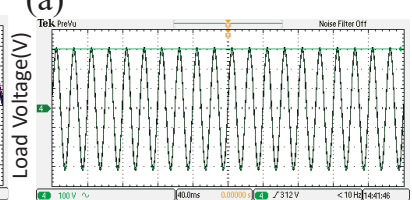

(c)
Fig. 14. Transient response of ratio changes from 1:1:1 to 1:1:2. (a) Instantaneous active and reactive power.(b) Output current of inverter \#1,\#2 and \#3. (c) Load voltage.

\#2 is same as inverter \#1 with $\left(0.5 V_{\text {rated }}, 0\right)$, and the oscillator initial state of inverter \#3 is $\left(0.9 V_{\text {rated }}, 0\right)$. Fig. 13(a)(b) illustrate active, reactive power and output current transient response, respectively. As it can be observed, the first and second connection settling time are approximately $0.6 \mathrm{~s}$ and $0.65 \mathrm{~s}$ respectively. Note that a small overshoot occur in the second connection due to the inverter \#3 has different initial state, so it is better to select the same initial states of inverters to eliminate extra overshoot in controller design processing. Moreover, the current transient also presents the rise-reduce dynamics with different initial states to affect the connection speed in Fig. 13 (c).

\section{B. Ratio Changes}

Next, we test the case for sudden current sharing ratio changes from 1:1:1 to 1:1:2 as shown in Fig. 14(a)-(c). Fig. 14(a) depicts the active and reactive power transient dynamics under ratio changes. Notice that the active power of inverter \#3 and inverters \#1 and \#2 separate at sudden changes. Reactive power present small transient then returning to 0 , and transient response only last $0.18 \mathrm{~s}$. Fig. 14(b) illustrates the output current of inverter \#3 increases immediately according to the sharing ratio $k$ changes. Meanwhile, the load voltage keeps stable when the ratio changes in Fig. 14(c).

\section{Nonlinear Load}

Here, we focus on the case of supplying a nonlinear load and removal one inverter from parallel system. Fig. 15(a) shows the power dynamics when inverter \#2 is removed from the system while supplying rectifier loads. Notice that there is a undershoot of reactive power under the removal due to the sudden decrease of the reactive current. Fig. 15(b) illustrates
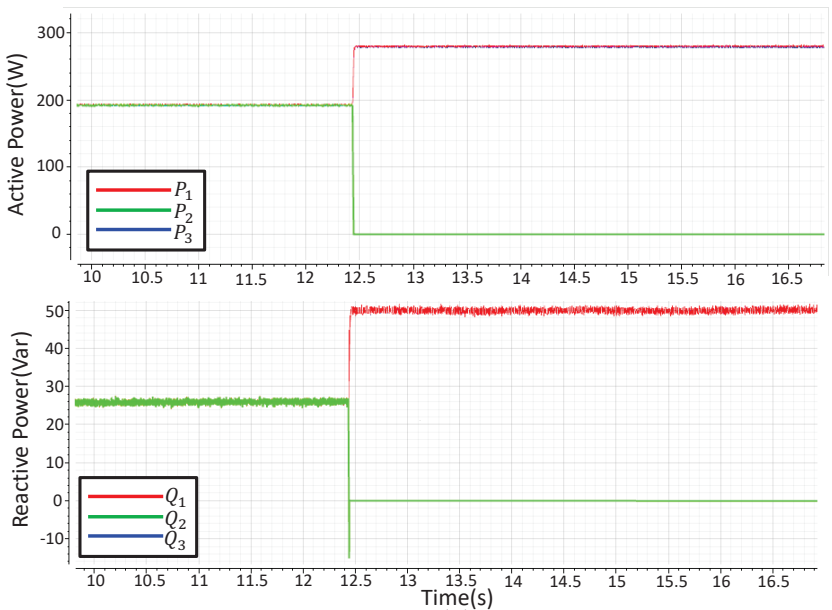

(a)

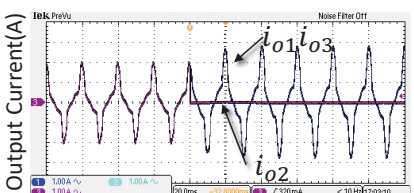

(b)

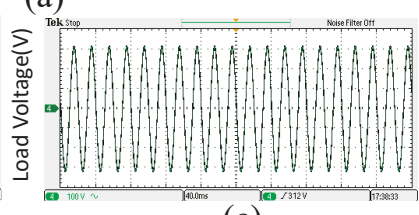

(c)
Fig. 15. Transient response of inverter removal with supplying a nonlinear load. (a) Instantaneous active and reactive power.(b) Output current of inverter \#1,\#2 and \#3. (c) Load voltage.
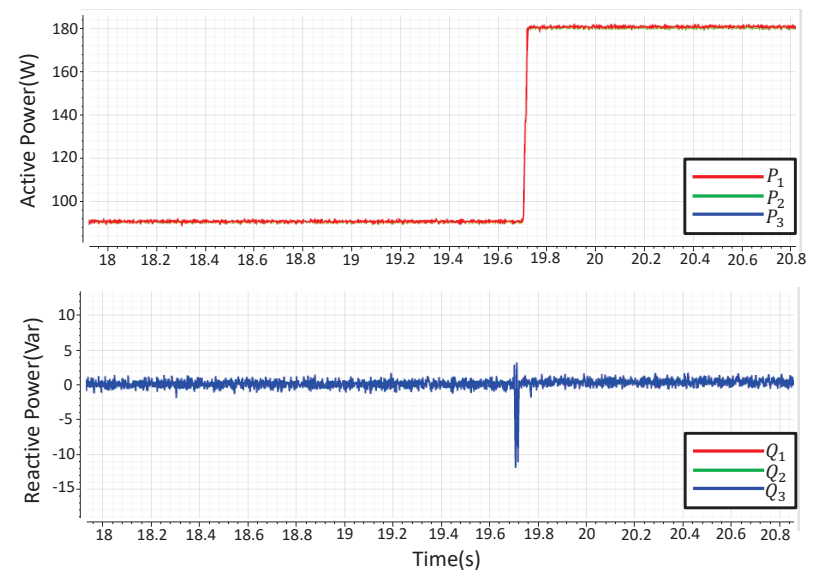

(a)

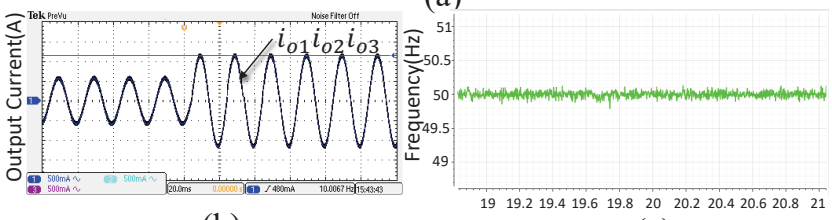

(b)

(c)

Fig. 16. Transient response of resistive load step. (a) Instantaneous active and reactive power.(b) Output current of inverter \#1,\#2 and \#3. (c) Load voltage frequency.

that inverter \#2 current suddenly decreases while the inverter $\# 1$ and \#3 current increase to supply the load persistently. The waveforms of load voltage keeps stable with slightly distortion as shown in Fig. 15(c). 


\section{Step Change}

Fig. 16(a)-(c) show the transient response during the load step changing when three parallelled inverters supply the common loads. We set the three inverters operate with the same current ratio, and then an extra $180 \Omega$ resistive load is parallel connected into the common bus. It is obvious to see that all three currents increase immediately to supply the common loads and the transient is minimal as shown in Fig. 16(b). The frequency response to loads step changes is presented in Fig. 16(c), which demonstrates the frequency deviation is small and keeps in the acceptable range, e.g. \pm 0.5 $\mathrm{Hz}$.

Consequently, the experimental validation results reveal the proposed Hopf-oscillator controller is able to realize the synchronization in parallel inverters system operation. The proposed strategy works well by fast response speed not only on the inverter connection and removal but also the sharing ratio changes case with resistive load or nonlinear load. Thus, the results also indicate that the proposed Hopf-oscillator controller performs as desired with load step.

\section{CONCLUSIONS}

This paper presented a simple and fast synchronization method based on coupled Hopf-oscillators for parallel singlephase inverters without communication in islanding microgrid. To compare with the Van der pol oscillator, the Hopf oscillator demonstrates the better robustness, the faster response, and the firm limit cycle in physical features. Therefore, the parallel inverters that are able to controlled as coupled Hopf oscillators to realize the synchronization. The steady-state model analyses clarify the relationship between the parameters and system performance. The global asymptotic synchronization proof indicates the sufficient condition for coupled oscillators system. A series of simulations and experiments were carried out to validate the proposed strategy. The obtained results do not require the power calculation and PLL, and present fast and precise synchronization and current sharing performance. As the part of future work, the oscillator controller for threephase inverter application is needed to realize in static threephase coordinates. Thus, the control strategy will be extended to gird-connected mode to complete the microgrid application. Therefore, the future effort can be focused on the analysis of robustness margins and power reference entry.

\section{REFERENCES}

[1] J. M. Guerrero, M. Chandorkar, T.-L. Lee, and P. C. Loh, "Advanced control architectures for intelligent microgirds part i: Decentralized and hierarchical control," IEEE Transactions on Industrial Electronics, vol. 60, no. 4, pp. 1254-1262, 2012.

[2] J. M. Guerrero, J. Matas, L. G. D. V. De Vicuna, M. Castilla, and J. Miret, "Wireless-control strategy for parallel operation of distributedgeneration inverters," IEEE Transactions on Industrial Electronics, vol. 53, no. 5, pp. 1461-1470, 2006.

[3] J. Rocabert, A. Luna, F. Blaabjerg, and P. Rodriguez, "Control of power converters in ac microgrids," IEEE transactions on power electronics, vol. 27, no. 11, pp. 4734-4749, 2012.

[4] Y. Guan, J. M. Guerrero, X. Zhao, J. C. Vasquez, and X. Guo, "A new way of controlling parallel-connected inverters by using synchronous reference frame virtual impedance loop part i: Control principle," IEEE Transactions on Power Electronics, vol. 31, no. 6, pp. 4576-4593, 2015.
[5] M. A. Schwemmer and T. J. Lewis, "The theory of weakly coupled oscillators," in Phase response curves in neuroscience, pp. 3-31. Springer, 2012.

[6] I. Szatmári and L. O. Chua, "Awakening dynamics via passive coupling and synchronization mechanism in oscillatory cellular neural/nonlinear networks," International Journal of Circuit Theory and Applications, vol. 36, no. 5-6, pp. 525-553, 2008.

[7] K. Chen and D. Wang, "A dynamically coupled neural oscillator network for image segmentation," Neural Networks, vol. 15, no. 3, pp. 423-439, 2002.

[8] C. M. Pinto and M. Golubitsky, "Central pattern generators for bipedal locomotion," Journal of mathematical biology, vol. 53, no. 3, pp. 474489, 2006.

[9] B. B. Johnson, S. V. Dhople, A. O. Hamadeh, and P. T. Krein, "Synchronization of parallel single-phase inverters with virtual oscillator control," IEEE Transactions on Power Electronics, vol. 29, no. 11, pp. 6124-6138, 2013

[10] B. B. Johnson, S. V. Dhople, A. O. Hamadeh, and P. T. Krein, "Synchronization of nonlinear oscillators in an lti electrical power network," IEEE Transactions on Circuits and Systems I: Regular Papers, vol. 61, no. 3, pp. 834-844, 2014.

[11] B. B. Johnson, M. Sinha, N. G. Ainsworth, F. Dörfler, and S. V. Dhople, "Synthesizing virtual oscillators to control islanded inverters," IEEE Transactions on Power Electronics, vol. 31, no. 8, pp. 6002-6015, 2015.

[12] B. B. Johnson, S. V. Dhople, J. L. Cale, A. O. Hamadeh, and P. T. Krein, "Oscillator-based inverter control for islanded three-phase microgrids," IEEE Journal of Photovoltaics, vol. 4, no. 1, pp. 387-395, 2013.

[13] M. Sinha, S. Dhople, B. Johnson, N. Ainsworth, and F. Dörfler, "Nonlinear supersets to droop control," in 2015 IEEE 16th Workshop on Control and Modeling for Power Electronics (COMPEL), pp. 1-6. IEEE, 2015.

[14] M. Sinha, F. Dörfler, B. B. Johnson, and S. V. Dhople, "Uncovering droop control laws embedded within the nonlinear dynamics of van der pol oscillators," IEEE Transactions on Control of Network Systems, vol. 4, no. 2, pp. 347-358, 2015.

[15] B. Johnson, M. Rodriguez, M. Sinha, and S. Dhople, "Comparison of virtual oscillator and droop control," in 2017 IEEE 18th Workshop on Control and Modeling for Power Electronics (COMPEL), pp. 1-6. IEEE, 2017.

[16] A. Pikovsky, J. Kurths, M. Rosenblum, and J. Kurths, Synchronization: a universal concept in nonlinear sciences, vol. 12. Cambridge university press, 2003.

[17] R. Grimshaw, Nonlinear ordinary differential equations. Routledge, 2017.

[18] D. Panayotounakos, N. Panayotounakou, and A. F. Vakakis, "On the lack of analytic solutions of the van der pol oscillator," ZAMM-Journal of Applied Mathematics and Mechanics/Zeitschrift für Angewandte Mathematik und Mechanik: Applied Mathematics and Mechanics, vol. 83, no. 9, pp. 611-615, 2003.

[19] H. Liu, W. Jia, and L. Bi, "Hopf oscillator based adaptive locomotion control for a bionic quadruped robot," in 2017 IEEE International Conference on Mechatronics and Automation (ICMA), pp. 949-954. IEEE, 2017.

[20] E. Oviedo, N. Vazquez, and R. Femat, "Synchronization technique of grid-connected power converters based on a limit cycle oscillator," IEEE Transactions on Industrial Electronics, vol. 65, no. 1, pp. 709-717, 2017.

[21] M. Lu, S. Dutta, V. Purba, S. Dhople, and B. Johnson, "A gridcompatible virtual oscillator controller: Analysis and design," in 2019 IEEE Energy Conversion Congress and Exposition (ECCE), pp. 26432649. IEEE.

[22] G.-S. Seo, M. Colombino, I. Subotic, B. Johnson, D. Groß, and F. Dörfler, "Dispatchable virtual oscillator control for decentralized inverter-dominated power systems: Analysis and experiments," in 2019 IEEE Applied Power Electronics Conference and Exposition (APEC), pp. 561-566. IEEE, 2019.

[23] M. Colombino, D. Groß, J.-S. Brouillon, and F. Dörfler, "Global phase and magnitude synchronization of coupled oscillators with application to the control of grid-forming power inverters," IEEE Transactions on Automatic Control, vol. 64, no. 11, pp. 4496-4511, 2019.

[24] B. D. Hassard, B. Hassard, N. D. Kazarinoff, Y.-H. Wan, and Y. W. Wan, Theory and applications of Hopf bifurcation, vol. 41. CUP Archive, 1981.

[25] M. Li, B. Wei, S. Tan, J. M. Guerrero, J. C. Vasquez et al., "Synchronization and current sharing for nonlinear-oscillator-based inverters in islanded three-phase microgrid," in 2019 IEEE Energy Conversion Congress and Exposition (ECCE), pp. 2566-2571. IEEE, 2019. 
[26] H. K. Khalil and J. W. Grizzle, Nonlinear systems, vol. 3. Prentice hall Upper Saddle River, NJ, 2002.

[27] S. Chen and Y. Cheung, "An elliptic lindstedt-poincaré method for certain strongly non-linear oscillators," Nonlinear Dynamics, vol. 12, no. 3, pp. 199-213, 1997.

[28] J. Grasman, F. Verhulst, and S.-D. Shih, "The lyapunov exponents of the van der pol oscillator," Mathematical methods in the applied sciences, vol. 28, no. 10, pp. 1131-1139, 2005.

[29] Y.-P. Choi, S.-Y. Ha, and S.-B. Yun, "Complete synchronization of kuramoto oscillators with finite inertia," Physica D: Nonlinear Phenomena, vol. 240, no. 1, pp. 32-44, 2011.

[30] M. G. Rosenblum, A. S. Pikovsky, and J. Kurths, "Phase synchronization of chaotic oscillators," Physical review letters, vol. 76, no. 11, p. 1804, 1996.

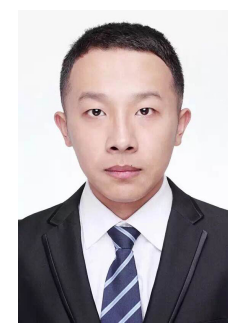

Mingshen Li (S'11) received the B.S. degree in electrical engineering from Chongqing University, Chongqing, China, in 2013, and the M.S. degree from the College of Electrical and Information Engineering, Hunan University, Changsha, China, in 2016. He is currently pursuing the Ph.D. degree with the Department of Energy Technology, Aalborg University, Aalborg, Denmark.

His current research interests include primary and distributed generation systems. control of converters, microgrid cluster systems,

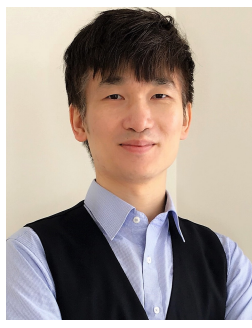

Yonghao Gui (S'11-M'17-SM'20) received the B.S. degree in automation from Northeastern University, Shenyang, China, in 2009, and the M.S. and Ph.D. degrees in electrical engineering from Hanyang University, Seoul, South Korea, in 2012 and 2017, respectively.

From Feb. 2017 to Nov. 2018, he worked with the Department of Energy Technology, Aalborg University, Aalborg, Denmark, as a Postdoctoral Researcher. Since Dec. 2018, he has been working with the Automation \& Control Section, Department of Electronic Systems, Aalborg University, Aalborg, Denmark, where he is currently an Assistant Professor. His research interests include Control of Power Electronics in Power Systems, Energy Internet, and Smart Grids.

Dr. Gui has served as an Associate Editor for the IEEE ACCESS and the International Journal of Control, Automation and Systems (IJCAS). He was a recipient of the IEEE Power \& Energy Society General Meeting Best Conference Paper Award in 2019 and the IJCAS Academic Activity Award 2019.

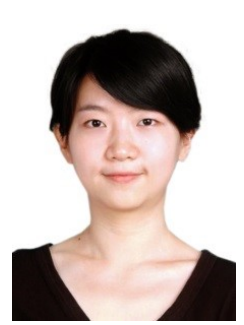

Yajuan Guan (S'14-M'16) received the B.S. degree and M.S. degree in electrical engineering from the Yanshan University, Qinhuangdao, China, and the Ph.D. degree in power electronics from the Aalborg University (AAU), Aalborg, Denmark, in 2007, 2010 and 2016 respectively. From 2010 to 2012, she was an Assistant Professor in Institute of Electrical Engineering (IEE), Chinese Academy of Sciences (CAS). In 2013, she was a Lecturer in IEE, CAS. From 2016 to 2018, she was a Postdoctoral Fellow with AAU. She is currently an Assistant Professor with AAU, as part of the Denmark Center for Research on Microgrids (https://www.crom.et.aau.dk/).

Her research interests include microgrids, distributed generation systems, power converters for renewable energy generation systems, and energy Internet.

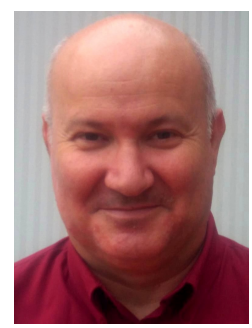

Jose Matas received the B.S., M.S., and Ph.D. degrees in telecommunications engineering from the Technical University of Catalonia, Barcelona, Spain, in 1988, 1996, and 2003, respectively. From 1988 to 1990 , he was an Engineer with a consumer electronics company. Since 1990 , he has been an Associate Professor with the Department of Electronic Engineering, Technical University of Catalonia, Spain. In 2017, he enrolled to the Department of Electrical Engineering.

His research interests include the areas of power electronics, nonlinear control, power quality, renewable energy systems, smart grid, and microgrids.

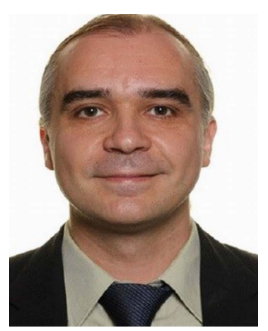

Josep M. Guerrero (S'01-M'04-SM'08-F'15) received the B.S. degree in telecommunications engineering, the M.S. degree in electronics engineering, and the Ph.D. degree in power electronics from the Technical University of Catalonia, Barcelona, Spain, in 1997, 2000, and 2003, respectively.

Since 2011, he has been a Full Professor with the Department of Energy Technology, Aalborg University, Aalborg, Denmark, where he is responsible for the Microgrid Research Program (www.microgrids.et.aau.dk). His research interest focuses on different microgrid aspects.

Dr. Guerrero was the recipient of the best paper award of the Journal of Power Electronics in 2016. In 2014, 2015, 2016, and 2017, he was awarded by T. Reuters as Highly Cited Researcher, and in 2015, he was elevated as the IEEE Fellow for his contributions on distributed power systems and microgrids.

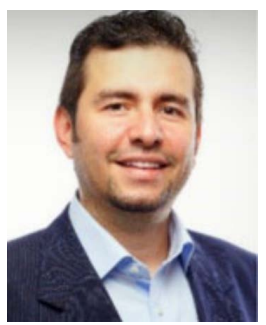

Juan C. Vasquez (M'12-SM'14) received the B.S. degree in electronics engineering from Universidad Autnoma de Manizales, Manizales, Colombia, in 2004, and the Ph.D. degree in automatic control, robotics, and computer vision from the Technical University of Catalonia, Barcelona, Spain, in 2009.

In 2011, he was an Assistant Professor with the Department of Energy Technology, Aalborg University, Aalborg, Denmark. Since 2018, he has been an Professor with the Department of Energy Technology, Aalborg University, where he is the Vice Programme Leader of the Microgrids Research Program. His current research interests include operation, advanced hierarchical and cooperative control, and the integration of Internet of Things into the Smart Grid.

Dr. Vasquez is an Associate Editor for the IET Power Electronics. In 2017, he was awarded by Thomson Reuters as a Highly Cited Researcher. He is currently a member of the IECSEG4 on LVDC safety for use in developed and developing economies, the TC-RES in the IEEE Industrial Electronics, PELS, IAS, and PES Societies. 